\title{
A dipole in a dielectric: Intriguing results and shape dependence of the distant electric field
}

\author{
R. L. P. G. Amaral and N. A. Lemos \\ Departamento de Física, Universidade Federal Fluminense, Av. Litorânea s/n, \\ Boa Viagem - CEP 24210-340, Niterói - Rio de Janeiro Brazil \\ The field of a point electric dipole in an infinite dielectric is obtained by placing \\ the dipole at the center of a spherical cavity of radius $R$ inside the dielectric and then \\ letting $R \rightarrow 0$. The result disagrees with the elementary answer found in textbooks. \\ The mathematical and physical reasons for the disagreement are discussed. The \\ discrepancy is confirmed by the same limiting procedure applied to a uniformly \\ polarized sphere embedded in the dielectric. We next solve the same problem for a \\ polarized spheroid immersed in an infinite dielectric and find that the asymptotic \\ potential shows an unexpected shape dependence, even after taking the limit of an \\ arbitrarily small spheroid. By considering both oblate and prolate spheroids and \\ taking appropriate limits, we recover either the elementary textbook answer or the \\ previous result found for the polarized sphere.
}

\section{INTRODUCTION}

Historically, electromagnetism, and particularly electrostatics, has been a rich source of beautiful mathematical physics problems, most of which are quite standard by now. Yet, from time to time, a closer look at certain simple and seemingly exhausted problems might

surprise even the experienced practitioner. We start by discussing the elementary problem of determining the electrostatic field produced by a pure (point) dipole embedded in the bulk of an infinite linear dielectric medium. This problem is solved by two apparently equivalent methods. The first makes use of an elementary argument found in textbooks and the other consists of putting the dipole at the center of a spherical hole in the dielectric and then letting the radius of the hole tend to zero. The discrepancy between the results might surprise the reader as much as it surprised the authors. The discrepancy is corroborated by the same limiting procedure applied to a uniformly polarized sphere embedded in the 
dielectric.

Next we solve for the electrostatic field of a uniformly polarized spheroid in an infinite dielectric. This solution is an interesting exercise in mathematical physics involving in a simple way Legendre functions of the second kind, which are seldom used in the standard electromagnetism textbooks. We find that the asymptotic potential exhibits a shape dependence. By taking appropriate limits, we recover either the elementary textbook answer or the previous result found for the polarized sphere. The dependence of the electrostatic potential on the shape of the spheroid, even after taking the limit in which the spheroid shrinks away keeping a finite dipole moment, is unexpected and to a certain extent non-intuitive. This physical effect appears to have been overlooked by standard textbooks.

\section{FIELD OF A DIPOLE IN A DIELECTRIC}

The problem of obtaining the field produced by a dipole in a dielectric medium is one of those elementary problems that is present (solved or proposed) in a variety of textbooks. The well-known solution is trivial. The physical dipole consists of two opposite point charges $\left(q\right.$ and $-q$ ) separated by the distance $d$. Letting $d \rightarrow 0$ with $q d=p_{0}$ gives the pure dipole. Because in a linear dielectric medium Gauss' law $\oint \mathbf{D} \cdot \mathbf{d a}=q$ establishes that each of the charges $q$ and $-q$ will be screened by polarization charges to $q^{\prime}=q \epsilon_{0} / \epsilon$, the dipole moment will be screened by the same factor, so that the actual (effective) dipole moment is

$$
p=p_{0} \frac{\epsilon_{0}}{\epsilon}
$$

Equation (1) is the answer found in standard textbooks (see Ref. 1 for example). In other words, for a point dipole parallel to the $z$-axis and located at the origin, the electrostatic potential inside the infinite linear dielectric medium in spherical coordinates $(r, \theta, \varphi)$ is

$$
\Phi(\mathbf{r})=\frac{p}{4 \pi \epsilon_{0}} \frac{\cos \theta}{r^{2}}
$$

with $p$ given by Eq. (11).

Now let us solve the "same" problem by putting the pure dipole $\mathbf{p}_{\mathbf{0}}$ at the center of an empty spherical hole of radius $R$ cut out of the dielectric medium and then letting $R \rightarrow 0$. It is appropriate to make use of the general solution to Laplace's equation in spherical coordinates for problems with azimuthal symmetry. It is easy to see that the boundary 
conditions can be satisfied by taking only the $\ell=1$ term of the azimuthally symmetric general solution, so the electrostatic potential inside the hole is

$$
\Phi^{(1)}(\mathbf{r})=A r \cos \theta+\frac{p_{0}}{4 \pi \epsilon_{0}} \frac{\cos \theta}{r^{2}}, \quad(0<r<R)
$$

and the potential outside is

$$
\Phi^{(2)}(\mathbf{r})=\frac{p^{\prime}}{4 \pi \epsilon_{0}} \frac{\cos \theta}{r^{2}} . \quad(r>R)
$$

Note that inside the hole the singular term corresponds to the pure dipole singularity with dipole moment $p_{0}$, because the dipole is in vacuum. Outside, only the term that decreases with $r$ is present, with the factor $p^{\prime}$ to be determined. By requiring the continuity of the scalar potential (equivalent to the continuity of the tangential component of the electric field) and of the radial component of the electric displacement vector $\left(\mathbf{D}^{(1)}=-\epsilon_{0} \nabla \Phi^{(1)}\right.$ and $\left.\mathbf{D}^{(2)}=-\epsilon \nabla \Phi^{(2)}\right)$ at the boundary $r=R$, we obtain

and

$$
-\left.\epsilon_{0} \frac{\partial \Phi^{(1)}}{\partial r}\right|_{r=R}=-\left.\epsilon \frac{\partial \Phi^{(2)}}{\partial r}\right|_{r=R}
$$

$$
\Phi^{(1)}(R)=\Phi^{(2)}(R)
$$

The application of the boundary conditions in Eqs. (田) and (5) leads to the equations

$$
\epsilon_{0}\left[\frac{2 p^{0}}{4 \pi \epsilon_{0} R^{3}}-A\right]=\epsilon \frac{2 p^{\prime}}{4 \pi \epsilon_{0} R^{3}},
$$

and

$$
\frac{p^{0}}{4 \pi \epsilon_{0} R^{2}}+A R=\frac{p^{\prime}}{4 \pi \epsilon_{0} R^{2}},
$$

whose solution is

$$
A=\frac{2\left(\epsilon_{0}-\epsilon\right)}{2 \epsilon+\epsilon_{0}} \frac{p_{0}}{4 \pi \epsilon_{0} R^{3}}
$$

and

$$
p^{\prime}=\frac{3 \epsilon_{0}}{2 \epsilon+\epsilon_{0}} p_{0} .
$$

According to Eqs. (8) and (9), the electrostatic potential outside the hole is that of a point dipole in vacuum with effective dipole moment $p^{\prime}$ given by Eq. (9). In the limit $R \rightarrow 0$, the dipole potential everywhere except at the origin is given by Eq. (3B) with $p^{\prime}$ determined by Eq. (9). Surprisingly, this effective dipole moment disagrees with the one given in Eq. (1) by means of the previous elementary argument. 
The reason for the discrepancy appears to be the lack of commutativity of two successive limits. The result (1) corresponds to putting the two opposite charges outside the hole in the dielectric, letting the radius of the hole tend to zero first, and then making the distance between the charges arbitrarily small, thus creating a dipole at the origin. To obtain the result (9), we first let the distance between the charges tend to zero, creating a point dipole at the center of the hole, and only later do we make the radius of the hole arbitrarily small. A physical explanation for the discrepancy is that in the first case, but not in the second case, the charges are always screened by the dielectric.

We might argue that the dipole moment associated with the polarization charges on the surface of the hole added to $p_{0}$ leads to a total dipole moment given by Eq. (9), which is in fact vindicated by an explicit calculation. This argument, however, misses the point. The surprise comes from the fact that, if only the free dipole moment $p_{0}$ is considered, its reduction by the dielectric constant factor does not account for the screening effect due to the polarization of the medium. This behavior contrasts sharply with that of a point charge at the center of the hole, whose field in the interior of the dielectric is obtained by simply replacing the free charge $q$ by $q \epsilon_{0} / \epsilon$ in the vacuum field.

\section{UNIFORMLY POLARIZED SPHERE IN A DIELECTRIC}

To check the previous result in Eq. (9) and allow for a generalization in Sec. IV, let us consider a uniformly polarized sphere (electret) of radius $R$, with polarization $\mathbf{P}$ along the $z$ axis, $\mathbf{P}=P_{0} \hat{\mathbf{k}}$, surrounded by an infinite dielectric whose dielectric constant is $\epsilon$. The potential has no singularity inside the sphere, so we have

$$
\Phi^{(1)}(\mathbf{r})=B r \cos \theta \quad(0<r<R)
$$

for the potential inside the sphere, while the potential outside is

$$
\Phi^{(2)}(\mathbf{r})=\frac{p^{\prime}}{4 \pi \epsilon_{0}} \frac{\cos \theta}{r^{2}} . \quad(r>R)
$$

We now notice that $\mathbf{D}^{(1)}=-\epsilon_{0} \nabla \Phi^{(1)}+\mathbf{P}$ and apply the same boundary conditions as before to obtain

$$
\frac{2 \epsilon p^{\prime}}{4 \pi \epsilon_{0} R^{3}}=-\epsilon_{0} B+P_{0}
$$

and 


$$
\frac{p^{\prime}}{4 \pi \epsilon_{0} R^{3}}=B
$$

which are solved by

$$
B=\frac{P_{0}}{2 \epsilon+\epsilon_{0}}
$$

and

$$
p^{\prime}=\frac{4 \pi \epsilon_{0}}{2 \epsilon+\epsilon_{0}} R^{3} P_{0}
$$

The resulting electrostatic potential inside the polarized sphere is

$$
\Phi^{(1)}(\mathbf{r})=\frac{P_{0}}{2 \epsilon+\epsilon_{0}} r \cos \theta, \quad(0<r<R)
$$

and the potential outside is

$$
\Phi^{(2)}(\mathbf{r})=\frac{R^{3} P_{0}}{2 \epsilon+\epsilon_{0}} \frac{\cos \theta}{r^{2}} . \quad(r>R)
$$

If we let $R \rightarrow 0$ and $P_{0} \rightarrow \infty$ in such a way that $p_{0}=(4 / 3) \pi R^{3} P_{0}$ remains fixed, we would expect to recover the point dipole $\mathbf{p}_{0}$ at the origin embedded in the infinite dielectric. In such a limit, the potential everywhere except at the origin becomes

$$
\Phi^{(2)}(\mathbf{r})=\frac{3 \epsilon_{0}}{2 \epsilon+\epsilon_{0}} \frac{1}{4 \pi \epsilon_{0}} \frac{p_{0} \cos \theta}{r^{2}} . \quad(r>0)
$$

This result coincides with the $R \rightarrow 0$ limit of the previous problem of the point dipole at the center of an empty sphere inside the dielectric.

Here, again, the dipole moment of the polarization charges on the spherical surface of the dielectric leads to the total dipole moment (91). Thus, the field inside the dielectric is

obtained from the vacuum field by reducing the free dipole moment by a factor that differs from the screening factor for a point charge.

\section{UNIFORMLY POLARIZED SPHEROID IN A DIELECTRIC}

To put the results of Sec. III in a broader context, which will make possible a further investigation of the origin of the discrepancy encountered above, we will examine a third "interpolating" problem. Consider a uniformly polarized hole (electret) in the dielectric medium with the shape of a spheroid (an ellipsoid of revolution). 


\section{A. The Oblate Case}

The oblate spheroidal coordinates are defined by (see Ref. 2 for example)

$$
\begin{aligned}
& x=a \cosh \mu \sin v \cos \varphi \\
& y=a \cosh \mu \sin v \sin \varphi \\
& z=a \sinh \mu \cos v,
\end{aligned}
$$

with $\mu \geq 0,0 \leq v \leq \pi, 0 \leq \varphi \leq 2 \pi$, and $a$ a positive real number. The surface of the spheroid is defined by $\mu=\mu_{0}$, while its interior is determined by $\mu<\mu_{0}$. It is easy to see that the surface of the spheroid is given in cartesian coordinates by

$$
\frac{x^{2}}{X^{2}}+\frac{y^{2}}{X^{2}}+\frac{z^{2}}{Z^{2}}=1
$$

where $X=a \cosh \mu_{0}$ and $Z=a \sinh \mu_{0}$, so that $X>Z$. The ellipsoid is oblate, that is, flattened along the $z$ direction.

In terms of the new variables

$$
\begin{aligned}
& \xi=\cos v \quad(-1 \leq \xi \leq 1) \\
& \zeta=\sinh \mu, \quad(0 \leq \zeta<\infty)
\end{aligned}
$$

we can write

$$
\begin{aligned}
& x=\rho \cos \varphi \\
& y=\rho \sin \varphi \\
& z=a \xi \zeta,
\end{aligned}
$$

with

$$
\rho=a\left[\left(1-\xi^{2}\right)\left(1+\zeta^{2}\right)\right]^{1 / 2}
$$

The surface of the spheroid is now given by $\zeta=\zeta_{0}$. Laplace's equation for the potential is separable in these coordinates, 2 and its solution with rotational symmetry about the $z$ axis, which is acceptable in the present physical circumstances, is

$$
\Phi^{(1)}(\xi, \zeta)=\sum_{\ell=0}^{\infty} P_{\ell}(\xi)\left[A_{\ell} P_{\ell}(i \zeta)+B_{\ell} Q_{\ell}(i \zeta)\right] \quad\left(\zeta<\zeta_{0}\right)
$$

inside the spheroid, and

$$
\Phi^{(2)}(\xi, \zeta)=\sum_{\ell=0}^{\infty} P_{\ell}(\xi)\left[C_{\ell} P_{\ell}(i \zeta)+D_{\ell} Q_{\ell}(i \zeta)\right] \quad\left(\zeta>\zeta_{0}\right)
$$


outside the spheroid, where $P_{\ell}$ is the $\ell$ th Legendre polynomial and $Q_{\ell}$ is the Legendre function of the second kind of order $\ell$. The absence of $Q_{\ell}(\xi)$ is necessary to guarantee the regularity of $\Phi$ on the $z$ axis $(\xi=1)$.

An inspection of Eq. (17) shows that asymptotically $\zeta$ plays the role of a radial coordinate. More precisely, for large $\mu$, we have $\xi \approx \cos \theta$ and $\zeta \approx r / a$ with $r, \theta$ spherical coordinates. This observation strongly suggests that the terms with $\ell=1$ alone will suffice to satisfy the boundary conditions, and accordingly we take

$$
\begin{array}{cc}
\Phi^{(1)}(\xi, \zeta)=P_{1}(\xi)\left[A P_{1}(i \zeta)+B Q_{1}(i \zeta)\right] \quad\left(\zeta<\zeta_{0}\right) \\
\Phi^{(2)}(\xi, \zeta)=P_{1}(\xi)\left[C P_{1}(i \zeta)+D Q_{1}(i \zeta)\right], \quad\left(\zeta>\zeta_{0}\right)
\end{array}
$$

where

$$
P_{1}(\xi)=\xi \quad \text { and } \quad Q_{1}(i \zeta)=\zeta \cot ^{-1} \zeta-1 .
$$

It is not difficult to show that for large $\zeta$

$$
Q_{1}(i \zeta) \longrightarrow-\frac{1}{3 \zeta^{2}}
$$

Therefore, the correct asymptotic behavior of $\Phi$ requires that $C=0$. As in the spherical coordinates case, it is necessary to take $B=0$ to avoid unphysical singularities. Indeed, the $\xi$-component of the electric field associated with the term $P_{1}(\xi) Q_{1}(i \zeta)$ is proportional to $h_{\xi}^{-1} \partial\left[P_{1}(\xi) Q_{1}(i \zeta)\right] / \partial \xi=a^{-1}\left(1-\xi^{2}\right)^{1 / 2}\left(\xi^{2}+\zeta^{2}\right)^{-1 / 2}\left(\zeta \cot ^{-1} \zeta-1\right)$, which is infinite at $\xi=\zeta=0$, that is, at the circumference $\rho=a$ on the $x y$-plane. Thus, we try to satisfy the boundary conditions with (the imaginary unit has been absorbed into the coefficient $A$ )

$$
\Phi^{(1)}(\xi, \zeta)=A \xi \zeta, \quad\left(\zeta<\zeta_{0}\right)
$$

and

$$
\Phi^{(2)}(\xi, \zeta)=D \xi\left(\zeta \cot ^{-1} \zeta-1\right) . \quad\left(\zeta>\zeta_{0}\right)
$$

The continuity of the potential at the surface of the spheroid yields

$$
A \zeta_{0}=D\left(\zeta_{0} \cot ^{-1} \zeta_{0}-1\right)
$$

The continuity of the normal component of $\mathbf{D}$ on the surface of the spheroid demands that

$$
-\left.\epsilon_{0} \frac{1}{h_{\zeta}} \frac{\partial \Phi^{(1)}}{\partial \zeta}\right|_{\zeta_{0}}+\left.\mathbf{P} \cdot \hat{\mathbf{e}}_{\zeta}\right|_{\zeta_{0}}=-\left.\epsilon \frac{1}{h_{\zeta}} \frac{\partial \Phi^{(2)}}{\partial \zeta}\right|_{\zeta_{0}},
$$


with

$$
h_{\zeta}=a\left(\frac{\xi^{2}+\zeta^{2}}{1+\zeta^{2}}\right)^{1 / 2}
$$

Because the unit outward normal vector to the surface of the spheroid is

$$
\hat{\mathbf{e}}_{\zeta}=\frac{\partial \mathbf{r} / \partial \zeta}{|\partial \mathbf{r} / \partial \zeta|}=\frac{1}{h_{\zeta}} \frac{\partial \mathbf{r}}{\partial \zeta}
$$

it follows that

$$
\mathbf{P} \cdot \hat{\mathbf{e}}_{\zeta}=P_{0} \hat{\mathbf{k}} \cdot \hat{\mathbf{e}}_{\zeta}=P_{0} \frac{1}{h_{\zeta}} \hat{\mathbf{k}} \cdot \frac{\partial \mathbf{r}}{\partial \zeta}=P_{0} \frac{a \xi}{h_{\zeta}} .
$$

The substitution of Eqs. (26) and (31) into Eq. (28) leads to

$$
-\epsilon_{0} A+P_{0} a=-\epsilon D\left(\cot ^{-1} \zeta_{0}-\frac{\zeta_{0}}{1+\zeta_{0}^{2}}\right) .
$$

The solution for $A$ and $D$ is

$$
\begin{aligned}
& A=\frac{\left(\cot ^{-1} \zeta_{0}-1 / \zeta_{0}\right) a P_{0}}{\left(\epsilon_{0}-\epsilon\right) \cot ^{-1} \zeta_{0}+\frac{\epsilon \zeta_{0}}{1+\zeta_{0}^{2}}-\frac{\epsilon_{0}}{\zeta_{0}}}, \\
& D=\frac{a P_{0}}{\left(\epsilon_{0}-\epsilon\right) \cot ^{-1} \zeta_{0}+\frac{\epsilon \zeta_{0}}{1+\zeta_{0}^{2}}-\frac{\epsilon_{0}}{\zeta_{0}}} .
\end{aligned}
$$

The volume of the spheroid is

$$
V=\frac{4}{3} \pi\left(a \cosh \mu_{0}\right)^{2} a \sinh \mu_{0}=\frac{4}{3} \pi a^{3}\left(1+\zeta_{0}^{2}\right) \zeta_{0},
$$

so that the dipole moment of the spheroid is

$$
p_{0}=\frac{4}{3} \pi a^{3}\left(1+\zeta_{0}^{2}\right) \zeta_{0} P_{0}
$$

For large $\zeta$, we have $\xi \approx \cos \theta$ and $\zeta \approx r / a$, so that, with the use of Eqs. (25) and (36), we find that the asymptotic behavior of the potential is

$$
\Phi^{(\mathrm{ob})}(\xi, \zeta) \longrightarrow \frac{\epsilon_{0}}{\left(\epsilon-\epsilon_{0}\right) \zeta_{0}\left(1+\zeta_{0}^{2}\right) \cot ^{-1} \zeta_{0}+\epsilon_{0}+\left(\epsilon_{0}-\epsilon\right) \zeta_{0}^{2}} \frac{p_{0}}{4 \pi \epsilon_{0}} \frac{\cos \theta}{r^{2}}
$$

This result is quite unexpected! Our intuition leads us to believe that seen from far away, it is impossible to tell a uniformly polarized sphere from a uniformly polarized ellipsoid. The shape independence of the asymptotic potential prevails only for the vacuum $\left(\epsilon=\epsilon_{0}\right)$. For a dielectric, the asymptotic potential depends on the shape of the dipole distribution near the origin. 


\section{B. The Prolate Case}

The analysis of the prolate case runs along similar lines. The prolate spheroidal coordinates are defined by

$$
\begin{aligned}
& x=a \sinh \mu \sin v \cos \varphi \\
& y=a \sinh \mu \sin v \sin \varphi \\
& z=a \cosh \mu \cos v .
\end{aligned}
$$

The surface of the spheroid is defined by $\mu=\mu_{0}$, while its interior is determined by $\mu<\mu_{0}$. The surface of the spheroid is again given in cartesian coordinates by Eq. (18) with $X=$ $a \sinh \mu_{0}$ and $Z=a \cosh \mu_{0}$, so that $Z>X$ and the spheroid is elongated in the $z$ direction.

In terms of the new variables

$$
\begin{aligned}
& \xi=\cos v \quad(-1 \leq \xi \leq 1) \\
& \eta=\cosh \mu, \quad(1 \leq \eta \leq \infty)
\end{aligned}
$$

we can write

$$
\begin{aligned}
& x=\rho \cos \varphi \\
& y=\rho \sin \varphi \\
& z=a \xi \eta,
\end{aligned}
$$

with

$$
\rho=a\left[\left(1-\xi^{2}\right)\left(\eta^{2}-1\right)\right]^{1 / 2} .
$$

The surface of the spheroid is now given by $\eta=\eta_{0}$, and the solution to Laplace's equation that suits our problem is

$$
\Phi^{(1)}(\xi, \eta)=F P_{1}(\xi) P_{1}(\eta), \quad\left(\eta<\eta_{0}\right)
$$

and

$$
\Phi^{(2)}(\xi, \eta)=G P_{1}(\xi) Q_{1}(\eta) . \quad\left(\eta>\eta_{0}\right)
$$

Here

$$
Q_{1}(\eta)=\frac{\eta}{2} \ln \frac{\eta+1}{\eta-1}-1,
$$

whose asymptotic behavior for large $\eta$ is

$$
Q_{1}(\eta) \longrightarrow \frac{1}{3 \eta^{2}}
$$


The application of the boundary conditions at the surface of the uniformly polarized prolate spheroid yields

$$
\begin{gathered}
F=\frac{\left(\frac{1}{2} \ln \frac{\eta_{0}+1}{\eta_{0}-1}-\frac{1}{\eta_{0}}\right) a P_{0}}{\frac{\epsilon_{0}-\epsilon}{2} \ln \frac{\eta_{0}+1}{\eta_{0}-1}+\frac{\epsilon \eta_{0}}{\eta_{0}^{2}-1}-\frac{\epsilon_{0}}{\eta_{0}}} \\
G=\frac{a P_{0}}{\frac{\epsilon_{0}-\epsilon}{2} \ln \frac{\eta_{0}+1}{\eta_{0}-1}+\frac{\epsilon \eta_{0}}{\eta_{0}^{2}-1}-\frac{\epsilon_{0}}{\eta_{0}}} .
\end{gathered}
$$

The volume of the prolate spheroid is

$$
V=\frac{4}{3} \pi\left(a \sinh \mu_{0}\right)^{2} a \cosh \mu_{0}=\frac{4}{3} \pi a^{3}\left(\eta_{0}^{2}-1\right) \eta_{0},
$$

and its dipole moment is

$$
p_{0}=\frac{4}{3} \pi a^{3}\left(\eta_{0}^{2}-1\right) \eta_{0} P_{0}
$$

As for the oblate case, we have for large $\eta, \xi \approx \cos \theta$ and $\eta \approx r / a$, so that the asymptotic behavior of the potential is

$$
\Phi^{(\mathrm{pr})}(\xi, \zeta) \longrightarrow \frac{\epsilon_{0}}{\frac{\epsilon_{0}-\epsilon}{2} \eta_{0}\left(\eta_{0}^{2}-1\right) \ln \frac{\eta_{0}+1}{\eta_{0}-1}+\epsilon_{0}+\left(\epsilon-\epsilon_{0}\right) \eta_{0}^{2}} \frac{p_{0}}{4 \pi \epsilon_{0}} \frac{\cos \theta}{r^{2}}
$$

Once again the asymptotic potential exhibits a surprising shape dependence that is absent only in the case of the vacuum. For a dielectric the asymptotic potential allows us to tell the difference between a uniform dipole density distributed within a sphere, an oblate spheroid, or a prolate spheroid. If a hole in the dielectric is filled with a uniform charge density, no such shape dependence is observed. The total induced charge depends only on the internal free charge and on the dielectric constant.

Note also the striking result that the electric field is uniform inside the spheroid because the potential is of the form $\Phi^{(1)}=A z$ in both cases, as Eqs. (26a) and (42) show.

\section{LIMITING CASES AND CONCLUSION}

Let us define the screening factor $\alpha$ as the coefficient that multiplies the vacuum asymptotic dipole field to give the asymptotic dipole field in the presence of the dielectric medium. From Eq. (50) it follows immediately that

$$
\alpha=\epsilon_{0}\left[\frac{\epsilon_{0}-\epsilon}{2} \eta_{0}\left(\eta_{0}^{2}-1\right) \ln \frac{\eta_{0}+1}{\eta_{0}-1}+\epsilon_{0}+\left(\epsilon-\epsilon_{0}\right) \eta_{0}^{2}\right]^{-1} .
$$


The spherical limit is reached by letting $\eta_{0} \equiv \cosh \mu_{0} \rightarrow \infty$ and $a \rightarrow 0$ in such a way that $a \cosh \mu_{0}=R$ remains fixed. Then we obtain $X=Z=R$ in Eq. (18) and the spheroid degenerates into a sphere. If we take into account that for large $\eta_{0}$

$$
\ln \frac{\eta_{0}+1}{\eta_{0}-1}=\frac{2}{\eta_{0}}+\frac{2}{3 \eta_{0}^{3}}+\cdots
$$

it is easy to show that

$$
\lim _{\eta_{0} \rightarrow \infty} \alpha=\frac{3 \epsilon_{0}}{2 \epsilon+\epsilon_{0}} .
$$

Thus our previous result for the uniformly polarized sphere is recovered.

Let us now examine the line dipole limit, reached by letting $\eta_{0} \rightarrow 1$ or, equivalently, $\mu_{0} \rightarrow 0$. In this limit we have $X=0$ and $Z=a$, so that the ellipsoid (18) reduces to a line segment (a rod) along the $z$-axis. If we recall that $\lim _{x \rightarrow 0} x \ln x=0$, we can readily show that

$$
\lim _{\eta_{0} \rightarrow 1} \alpha=\frac{\epsilon_{0}}{\epsilon},
$$

and the standard answer (1) is regained.

For the oblate spheroid we can also consider two limiting cases with the help of Eq. (37). If we let $\zeta_{0} \rightarrow \infty$ and $a \rightarrow 0$ with $a \zeta_{0}=R$, the spherical limit is reached and $\alpha \rightarrow 3 \epsilon_{0} /\left(2 \epsilon+\epsilon_{0}\right)$, as it should. If $\zeta_{0} \rightarrow 0$, the spheroid becomes a "pancake" describing a dipole layer, and $\alpha \rightarrow 1$. This result appears to be of some interest, inasmuch as the presence of the dielectric does not change the vacuum field.

We believe that further discussion of the physical grounds for the discrepancies is necessary. The screening factor $\alpha$ is a measure of the total dipole moment in the presence of the dieletric relative to the vacuum dipole moment $p_{0}$. Thus, the dipole moment of the charges induced on the surface of the hole depends not only on the free dipole moment $p_{0}$ and the dielectric constant, but also on the shape of the hole. That the shape dependence persists in the infinitely small hole limit seems to be related to the singularity of the dipole field, which is stronger than that of the monopole field. For a shrinking finite charge distribution (monopole), Gauss' law forbids this effect. The reader might want to generalize the dipole result for higher multipole moments.

It is suspected that such a shape dependence would manifest itself in the dynamical case, that is, in the radiation from a point dipole embedded in an infinite dielectric. Such a phenomenon might be of relevance in condensed matter physics. For instance, it might give rise to classical effects in the theory of quantum dots. 


\section{Acknowledgments}

This work was partially supported by Conselho Nacional de Desenvolvimento Científico e Tecnológico (CNPq), Brazil. Special thanks are due to David Griffiths, whose corrections and suggestions have contributed to a significant improvement of the paper. 
1 D. J. Griffiths, Introduction to Electrodynamics (Prentice Hall, New Jersey, 1999); see Problem 4.34 on p. 198 and let $R \rightarrow \infty$.

2 G. Arfken, Mathematical Methods for Physicists (Academic, New York, 1970), 2nd ed. 